\title{
REARRANGEMENTS AND FOURTH ORDER EQUATIONS
}

\author{
BY
}

VINCENZO FERONE (Dipartimento di Matematica e Applicazioni "R. Caccioppoli", Università di Napoli "Federico II", Complesso Monte S. Angelo, Via Cintia, 80126 Napoli, Italy)

AND

BERND KAWOHL (Mathematisches Institut, Universität zu Köln, D-50923 Köln, Germany)

\begin{abstract}
The paper contains a priori estimates for the deformation of plates and beams. In particular we investigate the "worst cases" for the maximum deformation depending on where a load is placed on a beam or plate. The methods of proof use rearrangement argument.
\end{abstract}

1. Introduction. A well known result for second order elliptic partial differential equations states that the solution $u$ of

$$
\begin{aligned}
-\Delta u=f & \text { in } \Omega, \\
u=0 & \text { on } \partial \Omega,
\end{aligned}
$$

can be compared to the solution $w$ of the symmetrized problem

$$
\begin{aligned}
-\Delta w & =f^{\#} & & \text { in } \Omega^{\#}, \\
w & =0 & & \text { on } \partial \Omega^{\#} .
\end{aligned}
$$

Here $\Omega^{\#}$ denotes a ball of same $n$-dimensional volume as $\Omega \subset \mathbb{R}^{n}$, and $f^{\#}$ denotes the spherically symmetric decreasing rearrangement (or Schwarz symmetrization) of $f$; see $[8,9]$. In fact, the pointwise estimate

$$
u^{\#}(x) \leq w(x) \text { for } x \in \Omega^{\#}
$$

is known to hold. This implies, in particular, that

$$
\|u\|_{L^{p}(\Omega)} \leq\|w\|_{L^{p}\left(\Omega \Omega^{\#}\right)} \text { for every } p \in[1, \infty] .
$$

Results of this nature are also known for quasilinear elliptic equations, parabolic equations, and for first order eikonal type equations.

In the present paper we attempt to derive similar results for fourth order equations. Rearrangement techniques can also be used to prove the Faber-Krahn inequality for the first eigenvalue of a clamped membrane. The analogous inequality for a clamped plate

Received October 22, 2001.

2000 Mathematics Subject Classification. Primary 35J40, 35Q75, 35B45, 74B05.

E-mail address: ferone@unina.it

E-mail address: kawohl@mi.uni-koeln.de 
is a much more formidable task, which has recently been solved in two and three space dimensions by Nadirashvili [7] and Ashbaugh and Benguria [1]. Here we investigate inhomogeneous equations instead of eigenvalue problems, and some of our results are stated only in one and two space dimensions. As a model equation we consider the plate equation

$$
\Delta^{2} u=f \quad \text { in } \Omega
$$

under various boundary conditions. There are Navier conditions $u=0=\Delta u$, which are prescribed for hinged beams or plates, Dirichlet conditions $u=0=\frac{\partial u}{\partial \nu}$, representing clamped beams or plates, and natural boundary conditions $\Delta u=0=\frac{\partial}{\partial \nu}(\Delta u)$ for free beams and plates. Let us list our results and interpret them.

THEOREM 1. For any natural $n$ compare the solution $u$ of

$$
\begin{aligned}
\Delta^{2} u & =f & & \text { in } \Omega, \\
\Delta u & =0=u & & \text { on } \partial \Omega .
\end{aligned}
$$

with the solution $v$ of

$$
\begin{aligned}
\Delta^{2} v & =f^{\#} & & \text { in } \Omega^{\#} \\
\Delta v & =0=v & & \text { on } \partial \Omega^{\#} .
\end{aligned}
$$

Then

$$
u^{\#}(x) \leq v(x) \text { for } x \in \Omega^{\#} .
$$

Theorem 1 says that the deformation of a hinged plate is maximal, if the plate is circular or a ball, and if the load is rearranged so that its maximum is centered at the center of the ball.

TheOREM 2. For $n=1$ compare the solution $u$ of

$$
u^{(4)}=f \quad \text { in }(0,1), \quad u(0)=u^{\prime}(0)=0=u^{\prime \prime}(1)=u^{\prime \prime \prime}(1),
$$

with the solution $v$ of

$$
v^{(4)}=f_{*} \quad \text { in }(0,1), \quad v(0)=v^{\prime}(0)=0=v^{\prime \prime}(1)=v^{\prime \prime \prime}(1),
$$

where $f_{*}$ denotes the monotone increasing rearrangement of $f$. Then

$$
|u(x)| \leq v(x) \text { for } x \in(0.1) .
$$

Moreover, if $f \geq 0$ in $(0,1)$, then $w \leq|u(x)|=u(x)$. Here $w$ solves

$$
w^{(4)}=f^{*} \quad \text { in }(0,1), \quad w(0)=w^{\prime}(0)=0=w^{\prime \prime}(1)=w^{\prime \prime \prime}(1),
$$

and $f^{*}$ denotes the monotone decreasing rearrangement of $f$.

Theorem 2 says that a cantilever beam, which is clamped at one end and free at the other end, undergoes maximum deformation if the load is concentrated at the free end and minimum deformation if the load is concentrated at the clamped end.

TheOREM 3. For $n=1$ compare the solution $u$ of

$$
u^{(4)}=f \geq 0 \quad \text { in }(-1,1), \quad u( \pm 1)=u^{\prime}( \pm 1)=0,
$$

with the solution $v$ of

$$
v^{(4)}=f^{\#} \quad \text { in }(-1,1), \quad v( \pm 1)=v^{\prime}( \pm 1)=0 .
$$


a) Then $\|u\|_{1} \leq\|v\|_{1}$ and $\|u\|_{\infty} \leq \frac{1}{24}\|f\|_{1}$. The last estimate becomes sharp as $f$ approaches $\delta_{0}(x)$.

b) If $f=\delta_{a}(x)$ for $a \in[0,1)$, then the solution is given by

$$
u_{a}(x)=\frac{1}{12}\left[|x-a|^{3}-(1-a x)^{3}+\frac{3}{2}(1-a x)\left(1-x^{2}\right)\left(1-a^{2}\right)\right]
$$

and $\left\|u_{a}\right\|_{\infty}=u_{a}\left(\frac{a}{a+2}\right)$ is decreasing in $a$. Its maximum is attained for $a=0$.

c) If $f=0.5$ is uniform, then $u(x)=\frac{1}{48}\left(1-|x|^{2}\right)^{2}$.

Theorem 3a says that the average deformation of a clamped beam becomes maximal when the load is centered around its center, and that the maximum deformation is attained when the total load is concentrated as a point-load in the center point. Theorem $3 \mathrm{~b}$ indicates that moving a point load towards the clamped boundary of the beam will diminish the maximum deformation. Theorem $3 \mathrm{c}$ shows that by distributing a total load uniformly, we can decrease the maximum deformation by one half, compared to the point load in the origin.

ThEOREM 4. For $n=2$ and $\Omega$ a disk of radius 1 consider the solution $u$ of

$$
\begin{aligned}
\Delta^{2} u & =f & & \text { in } \Omega, \\
\frac{\partial u}{\partial \nu} & =0=u & & \text { on } \partial \Omega .
\end{aligned}
$$

a) Then $\|u\|_{\infty} \leq \frac{1}{16 \pi}\|f\|_{1}$. This estimate becomes sharp as $f$ approaches $\delta_{0}(x)$.

b) If $f=\delta_{a}(x)$ and $|a| \in[0,1)$, then the solution is given by

$$
u_{a}(x)=\frac{1}{16 \pi}\left[\left(1-|a|^{2}\right)\left(1-|x|^{2}\right)-|x-a|^{2} \log \left(\frac{1+|a|^{2}|x|^{2}-2 a \cdot x}{|x-a|^{2}}\right)\right]
$$

and $\left\|u_{a}\right\|_{\infty}$ is decreasing in $|a|$. Its maximum is attained for $a=0$. Moreover,

$$
u_{a}(a)=\frac{1}{16 \pi}\left(1-|a|^{2}\right)^{2} \leq\left\|u_{a}\right\|_{\infty} \leq \frac{1}{16 \pi}\left(1-|a|^{2}\right)^{2} \frac{2}{|a|^{2}}\left(1-\sqrt{1-|a|^{2}}\right) .
$$

c) If $f=\frac{1}{\pi}$ is uniform, then $u(x)=\frac{1}{64 \pi}\left(1-|x|^{2}\right)^{2}$.

Theorem 4 reflects the fact that the deformation of a circular clamped plate becomes maximal, if the load is a point load which acts in the center. Moreover, according to Theorem $4 \mathrm{c}$ one can reduce this maximum by a factor of $\frac{1}{4}$ if the load is evenly distributed over the disk.

For the sake of completeness, before ending the section, we give the precise definition of the various types of rearrangements which have been used in the previous statements. For any measurable function $\varphi: \Omega \rightarrow \mathbb{R}$ we denote by $\mu_{\varphi}(t)$ the distribution function of $\varphi$ :

$$
\mu_{\varphi}(t)=|\{x \in \Omega:|\varphi(x)|>t\}|, \quad t \geq 0,
$$

where $|E|$ is the $n$-dimensional Lebesgue measure of a set $E$. The monotone decreasing rearrangement $\varphi^{*}$ of $\varphi$ is defined as the generalized inverse function of $\mu_{\varphi}$ :

$$
\varphi^{*}(s)=\inf \left\{t \in \mathbb{R}: \mu_{\varphi}(t) \leq s\right\}, \quad s \in(0,|\Omega|) .
$$


We recall that $\varphi$ and $\varphi^{*}$ are equimeasurable, i.e., $\mu_{\varphi}(t)=\mu_{\varphi^{*}}(t), \forall t \geq 0$. This implies, in particular, that

$$
\left\|\varphi^{*}\right\|_{L^{p}(0 .|S 2|)}=\|\varphi\|_{L^{\prime \prime}(\Omega 2)} . \quad \text { for every } p \in[1 . \propto] .
$$

The monotone increasing rearrangenent $\varphi_{*}$ and the spherically symmetric decreasing rearrangement $\varphi^{\#}$ of $\varphi$ are defined as follows:

$$
\begin{gathered}
\varphi_{*}(s)=\varphi^{*}(|\Omega|-s), \quad s \in(0 .|\Omega|) . \\
\varphi^{\#}(x)=\varphi^{*}\left(C_{n}|x|^{n}\right) . \quad x \in \Omega^{\#} .
\end{gathered}
$$

where $C_{n}$ denotes the unit ball in $\mathbb{R}^{n}$. By the definition it is clear that $\varphi_{*}, \varphi^{\#}$, and $\varphi$ are equimeasurable.

The theory of rearrangements is well known and exhaustive treatments of it can be found, for example, in $[2,6]$.

2. Proof of Theorem 1. To prove Theorem 1, we rewrite (1.3) as an elliptic system

$$
\begin{aligned}
-\Delta u & =\tilde{u} & & \text { in } \Omega . \\
-\Delta \tilde{u} & =f & & \text { in } \Omega . \\
u & =\tilde{u}=0 & & \text { on } \partial \Omega .
\end{aligned}
$$

and apply (1.2). Therefore $\tilde{u}^{\#}$ is dominated by $w$ from (1.1). If we apply (1.2) now to the first equation, $u^{\#}$ is dominated by the solution $z$ of

$$
-\Delta z=\tilde{u}^{\#} \quad \text { in } \Omega^{\#} . \quad z=0 \text { on } \partial \Omega^{\#} .
$$

The right-hand side in $(2.2)$ is dominated by $w$ and this coincides with $-\Delta v$, so $z \leq v$ in $\Omega^{\#}$ by the maximum principle. Therefore (1.5) is a consequence of $u^{\#} \leq z \leq v$.

3. Proof of Theorem 2. Using the boundary conditions at the free end we obtain

$$
u^{\prime \prime}(\zeta)=\int_{\zeta}^{1} \int_{\eta}^{1} f(\xi) d \xi d \eta \quad \text { for } \zeta \in[0,1] .
$$

Notice that for nommegative $f$. the function $u$ is convex and monotone increasing. Now we integrate from the clamped end and obtain

$$
u(x)=\int_{0}^{x} \int_{0}^{\omega} \int_{\zeta}^{1}\left[\int_{\eta}^{1} f(\xi) d \xi\right] d \eta d \zeta d \omega .
$$

This and

$$
\int_{\eta}^{1} f^{*}(\xi) d \xi \leq \int_{\eta}^{1}|f(\xi)| d \xi \leq \int_{\eta}^{1} f_{*}(\xi) d \xi
$$

imply Theorem 2 . 
4. Proof of Theorem 3. The solution $u$ of (1.10) can be represented in terms of the Green function, which is listed under (1.12) and taken from [3, p. 126; 5]. So

$$
u(x)=\int_{-1}^{1} u_{a}(x) f(a) d a \text { for } x \in(-1,1)
$$

and

$$
\|u\|_{1}=\int_{-1}^{1} \int_{-1}^{1} u_{a}(x) f(a) d a d x=\int_{-1}^{1}\left[\int_{-1}^{1} u_{a}(x) d x\right] f(a) d a .
$$

A simple integration gives $\int_{-1}^{1} u_{a}(x) d x=\frac{1}{24}\left(1-a^{2}\right)^{2}$, a symmetrically decreasing positive function of $a$. This proves the first part of statement a). To prove the second part of statement a) we recall (4.1) and see that $\|u\|_{\infty} \leq \sup _{a}\left\|u_{a}\right\|_{\infty}\|f\|_{1}$ and part b) give a).

To prove b) we look for zeroes of $u_{a}^{\prime}$, given $a>0$. There are zeroes at \pm 1 and at $\frac{a}{a+2}$. It is worth noting that the maximum deformation is not attained in $a$, the point where a point load is placed on the beam, but further inwards. Now a straightforward calculation gives

$$
\left\|u_{a}\right\|_{\infty}=\frac{1}{6} \frac{\left(1-a^{2}\right)^{2}(1+a)}{(a+2)^{2}},
$$

and the right-hand side of (4.1) is decreasing in $a \in(0.1)$, since its derivative equals

$$
\frac{a(1+a)^{2}(a+3)}{2(a+2)^{3}}(a-1)<0 .
$$

The proof of c) follows from a couple of elementary integrations.

5. Proof of Theorem 4. As in the previous theorem, the proof of statement a) follows from b) due to the representation formula

$$
u(x)=\int_{\Omega} u_{a}(x) f(a) d a \quad \text { for }|x| \in[0,1) .
$$

This time, however, $u_{a}$ is given by (1.14) and taken from [3, p. 126; 5].

To prove statement $b$ ), we abuse notation slightly. It suffices to consider the case that the point load attacks in $\mathbf{a}=(a, 0)$ with $a \in[0,1)$; i.e., a lies on the positive $x_{1}$-axis. Clearly we have $|\mathbf{a}|=a$.

In order to evaluate $\left\|u_{a}\right\|_{\infty}$ we compute partial derivatives of $u_{a}$ :

$$
\begin{aligned}
& \frac{\partial u_{a}}{\partial x_{1}}=\frac{1}{8 \pi}\left[-\frac{a\left(1-a^{2}\right)\left(1-a x_{1}\right)\left(1-|x|^{2}\right)}{1+a^{2}|x|^{2}-2 a x_{1}}+\left(a-x_{1}\right) \log \left(\frac{1+a^{2}|x|^{2}-2 a x_{1}}{|x|^{2}+a^{2}-2 a x_{1}}\right)\right], \\
& \frac{\partial u_{a}}{\partial x_{2}}=\frac{x_{2}}{8 \pi}\left[\frac{a^{2}\left(1-a^{2}\right)\left(1-|x|^{2}\right)}{1+a^{2}|x|^{2}-2 a x_{1}}+\log \left(1-\frac{\left(1-a^{2}\right)\left(1-|x|^{2}\right)}{1+a^{2}|x|^{2}-2 a x_{1}}\right)\right] .
\end{aligned}
$$

Taking into account the fact that

$$
\log (1-t)<-t, \quad \forall t \in(0,1),
$$

one sees that $x_{2} \frac{\partial u_{a}}{\partial x_{2}} \leq 0$ in $\Omega$ and that $\frac{\partial u_{a}}{\partial x_{2}}$ vanishes if and only if $x_{2}=0$ or $|x|=1$. This means that the maximum of $u_{a}$ has to be achieved along the $x_{2}$-axis in those points 
$\left(x_{1} .0\right)$ with $x_{1} \in(-1,1)$. for which

$$
\begin{aligned}
h_{a}\left(x_{1}\right) & \equiv \frac{\partial u_{a}}{\partial x_{1}}\left(x_{1}, 0\right) \\
& =\frac{1}{8 \pi}\left[-\frac{a\left(1-a^{2}\right)\left(1-x_{1}^{2}\right)}{1-a x_{1}}+\left(a-x_{1}\right) \log \left(\left(\frac{1-a x_{1}}{a-x_{1}}\right)^{2}\right)\right]=0 .
\end{aligned}
$$

We have

$$
h_{a}^{\prime \prime}\left(x_{1}\right)=-\frac{\left(1-a^{2}\right)^{3}}{4 \pi\left(1-a x_{1}\right)^{3}\left(a-x_{1}\right)} .
$$

i.e., $h_{a}$ is concave in $(-1, a)$ and convex in $(a, 1)$ and it vanishes in \pm 1 . Thus for any $a \in[0,1)$, the function $h_{a}$ has only one non-trivial zero in a point $x_{a} \in(-1,1)$. In such a point $u_{a}$ attains its maximum, i.c., $\left\|u_{a}\right\|_{\infty}=u_{a}\left(x_{a} .0\right)$. Furthermore $\frac{\partial u_{a}}{\partial x_{1}}\left(x_{1}, 0\right)>0$ for $x_{1} \in\left(-1, x_{a}\right)$ and $\frac{\partial u_{a}}{\partial x_{1}}\left(x_{1}, 0\right)<0$ for $x_{1} \in\left(x_{a}, 1\right)$. Incidentally, it is worth noting that the point $x_{a}$ lies closer to the origin than the point $(a, 0)$, in which the load is placed. Indeed, the fact that $\frac{\partial u_{a}}{\partial x_{1}}(0,0)=\frac{a}{8 \pi}\left(-\left(1-a^{2}\right)-\log \left(a^{2}\right)\right) \leq 0$ and $\frac{\partial u_{a}}{\partial x_{1}}(a, 0)=-\frac{a\left(1-a^{2}\right)}{8 \pi} \geq 0$ implies that $x_{a} \in[0, a]$ and that $x_{a}=a$ if and only if $a=0$.

Taking into account the fact that $x_{a}$ solves (5.3), one can compute the derivative of $\left\|u_{a}\right\| \infty$ with respect to $a$ obtaining:

$$
\frac{\mathrm{d}}{\mathrm{d} a}\left\|u_{a}\right\|_{\infty}=-\frac{\left(x_{a}+a\right)\left(1-a^{2}\right)\left(1-x_{a}^{2}\right)}{8 \pi\left(1-a x_{a}\right)} .
$$

This means that $\left\|u_{a}\right\|_{\infty}$ is decreasing with respect to $a$ and achieves its maximum for $a=0$.

The fact that $u(a, 0) \leq u\left(x_{a}, 0\right)$ proves the first inequality in (1.15). As regards the second inequality in (1.15). we observe that due to (5.3) one obtains

$$
u_{a}\left(x_{a}, 0\right)=\frac{\left(1-a^{2}\right)^{2}}{16 \pi} H\left(x_{a}\right), \quad \text { where } H(x)=\frac{1-x^{2}}{1-a x} .
$$

Notice that $H(x) \geq 1$ for $x \in(0, a)$. Now we estimate $H\left(x_{a}\right)$ from above by the maximum of $H$ on $(0, a)$. It is attained in $\tilde{x}_{a}=\frac{1}{a}-\frac{1}{a} \sqrt{1-a^{2}}$ and $H\left(x_{a}\right) \leq H\left(\tilde{x}_{a}\right)=\frac{2}{a^{2}}\left(1-\sqrt{1-a^{2}}\right)$. This completes the proof of claim b).

Claim c) follows from a straightforward calculation.

\section{REFERENCES}

[1] M. S. Ashbaugh and R. D. Benguria, On Rayleigh's conjecture for the clamped plate and its generalization to three dimensions, Duke Math. J. 78 (1995) pp. 1-17.

[2] C. Bandle, Isoperimetric Inequalities and Applications. Monographs and Studies in Math., No. 7 , Pitman, London (1980).

[3] T. Boggio, Sulle funzioni di Green d'ordine m, Rend. Circ. Mat. Palermo 20 (1905), pp. 97-135.

[4] G. Buttazzo, M. Giaquinta and S. Hildebrandt, One-dimensional Variational Problems, Clarendon Press, Oxford (1998).

[5] H. C. Grunau and G. Sweers, Positivity for equations involving polyharmonic operators with Dirichlet boundary conditions, Math. Annalen 307 (1997), pp. 589-626.

[6] B. Kawohl, Rearrangements and convexity of level sets in P.D.E., Lecture Notes in Math., No. 1150, Springer, Berlin-New York (1985).

[7] N. S. Nadirashvili, Rayleigh's conjecture on the principal frequency of the clamped plate, Arch. Ration. Mech. Anal. 129 (1995). pp. 1-10. 
[8] G. Talenti, Elliptic equations and rearrangements, Ann. Sc. Norm. Sup. Pisa, Ser. IV, 3 (1976), pp. $697-718$.

[9] H. P. Weinberger, Symmetrization in uniformly elliptic problems, in: Studies in Math Analysis and Related Topics, Univ. of Calif. Press, Stanford, CA (1962), pp. 424-428. 